\title{
Cell carriers to deliver oncolytic viruses to sites of myeloma tumor growth
}

\author{
A Munguia, T Ota, T Miest and SJ Russell \\ Department of Molecular Medicine, Mayo Clinic, Rochester, MN, USA
}

\begin{abstract}
Multiple myeloma (MM) is a disseminated malignancy of antibody secreting plasma cells that localize primarily to the bone marrow. Several studies have illustrated the potential of utilizing oncolytic viruses (measles, vaccinia, Vesicular Stomatitis Virus and coxsackievirus A21) for the treatment of $M M$, but there are significant barriers that prevent the viruses from reaching sites of myeloma tumor growth after intravenous delivery. The most important barriers are failure to extravasate from tumor blood vessels, mislocalization of the viruses in liver and spleen and neutralization by antiviral antibodies. In this review, we discuss the use of various cell types as carriers to overcome these barriers, emphasizing their relative susceptibilities to virus infection and their variable trafficking properties. Mesenchymal progenitor cells, monocytes and $T$ cells have all shown promise as virus-delivery vehicles capable of accessing sites of
\end{abstract}

Keywords: cell carriers; oncolytic virotherapy; multiple myeloma myeloma growth. However, a previously unexplored alternative would be to use primary myeloma cells, or even myeloma cell lines, as delivery vehicles. Advantages of this approach are the natural ability of myeloma cells to home to sites of myeloma tumor growth and their compatibility with tumor-specific viruses that cannot propagate in other carrier cell lineages. A potential difficulty associated with the use of myeloma cells for virus delivery is that they must be exposed to supralethal doses of ionizing radiation before they can be safely administered to patients. Preliminary studies are presented in which we demonstrate the feasibility of using irradiated myeloma cells as carriers to deliver oncolytic viruses to sites of myeloma tumor growth in an orthotopic human myeloma model.

Gene Therapy (2008) 15, 797-806; doi:10.1038/gt.2008.45; published online 20 March 2008

\section{Multiple myeloma}

Multiple myeloma (MM) is a disseminated malignancy of antibody secreting plasma cells located predominately in the bone marrow. ${ }^{1}$ The disease responds initially to alkylating agents and corticosteroids, but eventually becomes refractory. High-dose (myeloablative) melphalan therapy followed by autologous stem cell transplantation increases the number of complete remissions but survival is not greatly prolonged..$^{2-4}$ Characteristically, the neoplastic cells also form discrete, well-vascularized tumors (skeletal plasmacytomas) that strongly stimulate osteoclastic bone resorption and erode into adjacent cortical bone. Clinical complications from myeloma include bone lesions with severe pain, hypercalcemia and renal dysfunction. ${ }^{5}$

The biology of MM has been extensively studied and it has been determined that chemokines in the microenvironment of the bone marrow are vital for the growth and maintenance of myeloma cells. ${ }^{6}$ The microenvironment of the bone marrow is comprised of osteoblasts, osteclasts, bone marrow stromal cells (BMSCs), extracellular matrix proteins, lymphocytes and endothelial

Correspondence: Dr SJ Russell, Department of Molecular Medicine, Mayo Clinic MN, Guggenheim 1821D 200, First Street SW, Rochester, Minnesota 55905, USA.

E-mail: sjr@mayo.edu

Received 14 February 2008; revised 18 February 2008; accepted 19 February 2008; published online 20 March 2008 cells. ${ }^{6}$ The homing of myeloma cells to the bone marrow is, in part, dependent upon their expression of the chemokine receptor CXCR4. The ligand for CXCR4 is the cytokine stromal cell-derived factor $1 \alpha$, also known as CXCL12, which is highly expressed in the bone marrow. ${ }^{7-9}$ Once in the bone marrow, the myeloma cells adhere to BMSCs, resulting in increased cytokine production from the myeloma cells, BMSCs and other cells to facilitate the proliferation, survival and drug resistance of myeloma cells., ${ }^{6,10-15}$ Some of the newer antimyeloma drugs have been shown to target the bone marrow microenvironment and hinder the spread of the myeloma cells. These include thalidomide, bortezomib and lenalidomide. ${ }^{16-19}$ Despite the availability of new treatments, MM remains incurable and causes more than 10000 deaths per year in the United States. ${ }^{20}$ Median survival is approximately 4 years and alternative approaches to therapy are urgently required. In this review, we discuss the use of oncolytic viruses as novel antimyeloma agents, and in particular we explore the possibility of utilizing cell carriers to deliver oncolytic virus to site of myeloma tumor growth.

\section{Oncolytic viruses for the treatment of MM}

Oncolytic viruses have been shown to have great potential for the treatment of cancer due to their high tropism for tumor cells. ${ }^{21,22}$ There are several oncolytic 
viruses that have shown promise for the treatment of MM and they are discussed below.

\section{Measles virus}

Measles virus is a negative sense single-stranded RNA virus belonging to the Paramyxoviridae family and Morbillivirus genus. The attenuated MV-Edm (Edmonston) strain is potentially oncolytic against several types of cancer. $^{23}$ A preliminary study illustrated that $\mathrm{MV}$ replicated efficiently in several myeloma cell lines and in myeloma cells removed from myeloma patients. In contrast, primary phytohemagglutinin-stimulated peripheral blood lymphocytes from healthy donors were not efficiently infected with $\mathrm{MV}-\mathrm{Edm}{ }^{24}$ Severe-combined immunodeficiency mice bearing subcutaneous myeloma xenografts were utilized to address the in vivo application of MV-Edm for the treatment of MM. There was complete tumor regression in severe-combined immunodeficiency mice that were treated with intratumoral injections of MV-Edm but not in tumor bearing mice that were treated with ultraviolet-inactivated MV-Edm. In addition, when multiple doses of MV-Edm were systemically administered to mice bearing myeloma xenografts, there was an increased survival rate of mice and increased frequency of complete tumor regression compared to mice that received a single dose of MVEdm or ultraviolet-inactivated virus.

CD46 and signaling lymphocyte activation molecule have been identified as MV receptors. ${ }^{25}$ Signaling lymphocyte activation molecule is expressed on T cells, $\mathrm{B}$ cells and monocytes, whereas CD46 is expressed on all human nucleated cells. ${ }^{26}$ Retargeting strategies have, therefore, been employed to increase the accuracy of MV infection of myeloma cells. MV-Edm was engineered such that the $\mathrm{H}$ protein displayed the single chain antibody (scFv) to CD38 (MV- $\propto \mathrm{CD} 38)$ at its extreme C-terminus. ${ }^{27}$ CD38 is a cell marker used to identify myeloma cells. $\mathrm{CHO}$ cells, which are naturally resistant to MV infection, that expressed CD38 were susceptible to MV- $\propto$ CD38 infection, but not to MV-Edm. However, virus retargeting does not appear to be necessary to ensure selective killing of myeloma cells.

Human tumor cells generally show an increase in CD46 expression compared to non-tumor cells. ${ }^{28}$ In addition, a strong correlative effect has been illustrated between increased CD46 expression and increased potency of MV-Edm oncolytic infection. ${ }^{29}$ Bone marrow samples collected from myeloma patients revealed a sevenfold increase in CD46 expression levels on myeloma cells compared to non-myeloma cells. In addition, the myeloma cells showed greatly increased susceptibility to MV infection, fusion and killing, which correlated to their increased expression of CD46. CD46 appeared to be the most important cell marker on myeloma cells determining their susceptibility to $\mathrm{MV}$ oncolytic infection. ${ }^{30}$

The human sodium-iodide symporter (hNIS) is a transmembrane ion symporter that concentrates iodide in thyroid follicular cells and is being evaluated as a therapeutic gene for the treatment for cancer. ${ }^{31,32}$ To facilitate the noninvasive monitoring of oncolytic MV and to enhance its oncolytic efficiency, the virus was engineered to express hNIS (MV-NIS). Radioiodine was concentrated in MV-NIS-infected cells and the concentration of radioiodine increased as the MV-NIS infection progressed. Additionally, when MV-NIS was administered via the bloodstream to mice bearing human myeloma xenografts, intratumoral spread of the virus could be monitored in a noninvasive manner with radioiodine. Furthermore, there was complete regression of a measles resistant myeloma tumor model when MVNIS was combined with a therapeutic dose of radioiodine in the form of ${ }^{131} \mathrm{I}^{33}$ MV-NIS is currently being administered intravenously to patients with MM in a phase I clinical trial at Mayo Clinic. ${ }^{34}$

\section{Vesicular stomatitis virus}

Vesicular stomatitis virus (VSV) is a negative sense single-stranded RNA virus belonging to the family Rhabdoviridae. VSV is highly sensitive to the antiviral effects of interferon system. Propagation of VSV is hindered in cells that contain a functional interferon signaling system. ${ }^{35}$ VSV has been shown to selectively infect and kill malignant cells more efficiently than nonmalignant cells, even in the presence of interferon. ${ }^{36}$ In addition, preliminary studies have shown that VSV is a potent oncolytic agent even when administered systemically. ${ }^{37,38}$ Lichty et al. ${ }^{39}$ illustrated that myeloma cell lines were susceptible to oncolytic infection by VSV and that bone marrow progenitor cells were resistant. VSV infection was also shown to decrease the percentage of CD138+ cells in blood samples collected from MM patients.

Building on this work, Goel et al. ${ }^{40}$ engineered VSV $\Delta 51$ to express the $h N I S$ gene (VSV $\Delta 51-\mathrm{hNIS}$ ) and examined the potential of VSV radiovirotherapy for the treatment of MM. VSV $\Delta 51$ has a deletion of methionine 51 in the matrix protein $(\mathrm{M})$ such that it cannot block the nuclear export of interferon RNA and, therefore, illustrates an increased antitumor specificity compared to VSV. ${ }^{36,41}$ Since murine cells are susceptible to VSV infection, in vivo studies were performed using a syngeneic 5TGM1 mouse myeloma model. Mice bearing subcutaneous 5TGM1 myeloma tumors showed tumor regression and an increased survival rate when VSVD51-hNIS was administered by intratumoral or intravenous injection. Furthermore, there was an increase in oncolytic activity when VSV $\Delta 51-\mathrm{hNIS}$ treatment was combined with a therapeutic dose of ${ }^{131} \mathrm{I}$. Intratumoral propagation of VSV $\Delta 51-\mathrm{hNIS}$ could be noninvasively monitored with radioiodine imaging. Thus, VSV $\Delta 51-h N I S$ showed great potential as a novel radiovirotherapy agent for the treatment of MM.

\section{Coxsackievirus A21}

CVA21, a member of the Picornaviridae family, is a nonenveloped, positive sense single-stranded RNA virus that is known to cause the 'common cold'. ${ }^{42}$ Recent studies have illustrated that CVA21 is potentially oncolytic for the treatment of human melanoma, both in vitro and in vivo. ${ }^{43,44}$ Intercellular adhesion molecule-1 and decay-accelerating factor are both necessary for CVA21 attachment and subsequent virus infection leading to cell lysis. ${ }^{45} \mathrm{Au}$ et al. ${ }^{46}$ recently reported that there is an increase in the expression of both intercellular adhesion molecule-1 and decay-accelerating factor in MM cell lines and bone marrow biopsies obtained from MM patients. The MM cells were susceptible to CVA21 infection and could produce progeny CVA21. In contrast, peripheral blood mononuclear cells expressed low levels 
of intercellular adhesion molecule-1 and were not efficiently infected with CVA21. Ex vivo CVA21 infection of BM samples from MM patients at various stages of their disease illustrated that the CVA21 was selectively killing the CD138+ myeloma cells, but not CD138- cells. These results do illustrate the potential of CVA21 to serve as an oncolytic agent for the treatment of MM. In addition, in vivo experiments that were recently conducted in our laboratory have demonstrated that CVA21 is potentially oncolytic in mouse xenograft models of myeloma (E Kelly et al., unpublished data).

\section{Vaccinia virus}

A case study involving a 67-year-old Japanese male with a diagnosis of $\operatorname{IgA}$ MM was one of the first cases that described the possibility of using oncolytic viruses for the treatment of $\mathrm{MM}^{4}{ }^{4}$ In this particular study, the patient was treated with attenuated vaccinia virus AS (AS) strain. Studies prior to this had shown that the AS strain was active in the treatment of two patients with adenocarcinoma with minimal adverse side effects. ${ }^{48}$ The myeloma patient received multiple doses of the AS strain over the period of 12 weeks via the intravenous route. On the 15th day after initiation of treatment, the serum IgA levels started to decrease and on the 96th post-treatment day the serum IgA level was $432 \mathrm{mg}$ per $100 \mathrm{ml}$ compared to $1309 \mathrm{mg}$ per $100 \mathrm{ml}$ at the beginning of treatment. The patient did not illustrate any apparent adverse effects to the AS strain treatments.

\section{Barriers to oncolytic virotherapy for the treatment of MM}

For the treatment of MM, the oncolytic viruses must be delivered via the bloodstream. Once the oncolytic virus reaches the tumor site, it must accurately and efficiently recognize and destroy the tumor tissue. There are many barriers that impede the delivery of 'naked' virions injected via the bloodstream. Of these, the three most significant are (i) circulating antiviral antibodies (ii) nonspecific uptake of virus by liver, spleen and other non-malignant tissues, and (iii) poor extravasation of the virus from the blood vessels to the tumor site. All three of these barriers are potentially surmountable by using cell carriers to deliver the virus to the tumor.

\section{Utilization of cell carriers to circumvent virotherapy barriers}

Cells are rapidly gaining popularity as delivery vehicles for genes and viruses. An assortment of cells have been explored in this regard, including tumor cells, ${ }^{49-52}$ outgrowth endothelial cells, ${ }^{53}$ mesenchymal progenitor cells, ${ }^{54,55} \mathrm{~T}$ cells ${ }^{56,57}$ and monocytes. ${ }^{58}$ There are several criteria that need to be addressed when determining which cell type should be utilized as a carrier for the delivery of a given oncolytic virus: (i) susceptibility to oncolytic virus infection, (ii) protection of the cargo from antibody neutralization, (iii) homing to sites of tumor growth and (iv) transfer of virus progeny to tumor tissue.

\section{Cell carriers to deliver oncolytic virus to sites of myeloma tumor growth}

For clinical applications in patients with MM, it is important to focus on cells that can be prepared in large numbers. Since myeloma cells reside predominately in the bone marrow, mesenchymal progenitor cells, which traffic to the bone marrow, should be considered as carriers to deliver oncolytic viruses to sites of myeloma growth. Recently, we examined plasmacytomas from MM patients and found that they were uniformly infiltrated with macrophages and partially infiltrated with T lymphocytes (KW Peng et al., unpublished data).

\section{Cell Carriers for Myeloma Virotherapy}

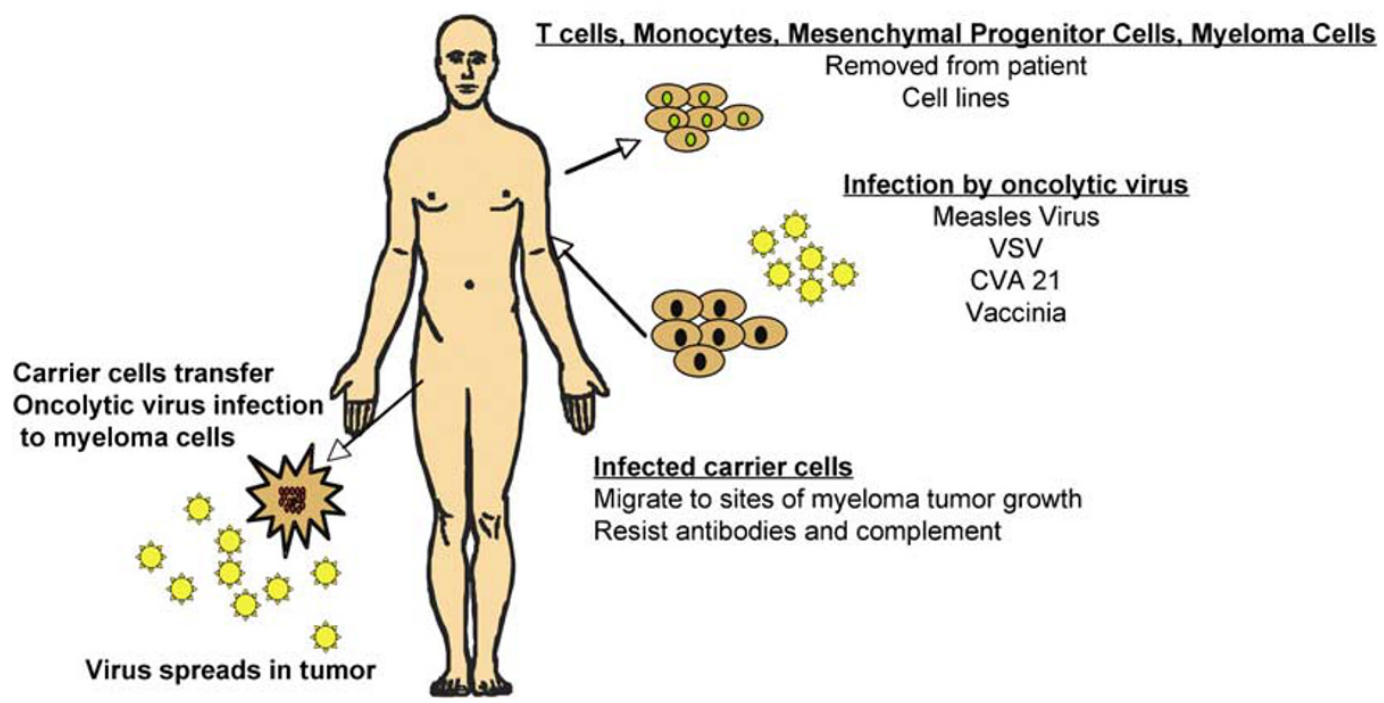

Figure 1 Potential cell carriers for myeloma virotherapy. Schematic diagram illustrating how cell carriers could be utilized to deliver oncolytic virus to the sites of myeloma tumor growth. Since myeloma cells reside in the bone marrow, potential cell carriers should be able to traffic to the bone marrow. Possibilities include T cells, monocytes, mesenchymal stem cells and myeloma cells, all of which may be autologous or allogeneic. 
These findings suggested that ideal cell carriers for myeloma virotherapy would be mesenchymal progenitor cells, monocytes, T lymphocytes or myeloma cells themselves (Figure 1). The potential cell carriers could be autologous or allogeneic.

\section{Mesenchymal progenitor cells}

Mesenchymal progenitor cells originate in the bone marrow and can differentiate into a variety of cell types that localize to sites of injured tissue. ${ }^{59,60}$ One study has shown that adenovirus-infected MPCs have tumorhoming capabilities after local or systemic administration. ${ }^{54,55}$ A major advantage of MPCs is that they are readily available from healthy donors and can be expanded quickly in cell culture. ${ }^{61}$ Therefore, it can be suggested that MPCs have the potential to serve as cell carriers for the treatment of MM, since these cells are derived from the bone marrow and travel via the blood vessels.

\section{Monocytic lineage}

Monocytes and their progeny express a variety of adhesive and signaling receptors whose patterns and levels of expression vary over time, with differentiation and changes in activation status. ${ }^{62}$ Monocytes can interact with vascular endothelial cells, migrate out of blood vessels into tumors and other tissues, and differentiate into phagocytic macrophages or dendritic cells. ${ }^{63}$ As monocytes mature to become dendritic cells, they increase their expression of the lymph node-homing receptor, CCR7. Dendritic cell homing is not fully understood and there could be other adhesion and chemokine receptors beside CCR7 that mediate this trafficking. In addition, monocyte-derived cells can form synapses with heterologous cells, where adhesive interactions lead to an intimate association between adjacent cell membranes. ${ }^{64}$ Therefore, it can be hypothesized that this interaction between infected and uninfected cells will provide a protected microenvironment, which will render viral glycoproteins inaccessible to circulating antibodies. Recently, we illustrated that a monocytic cell line infected with MV could 'bypass' the inhibitory effects of MV antibodies and deliver MV to sites of tumor growth. ${ }^{58}$

Macrophages are monocyte-derived cells that are often found in abundance in tumors and can be categorized as either M1 or M2 subtypes. ${ }^{65}$ M1 macrophages suppress tumor growth, while M2 macrophages promote tumor growth. Tumor-associated macrophages are usually M2 macrophages which support the proliferation and survival of tumors by releasing growth factors that promote angiogenesis and tumor matrix remodeling. ${ }^{66}$ Many tumors release monocytic chemotactic factors such as monocyte chemoattractant protein-1 (MCP-1), also known as CCL2, macrophage colony stimulating factor (M-CSF), and vascular endothelial growth factor (VEGF). Once monocytes have been recruited to the tumor microenvironment, they differentiate into tumor-associated macrophages. As mentioned previously, the interaction of myeloma cells with BMSCs leads to the release of cytokines that promote myeloma growth progression. The secretion of VEGF by myeloma cells may stimulate IL-6 secretion from BMSCs and both of these cytokines promote myeloma growth. ${ }^{67}$ In addition, IL-6 promotes the increased release of MCP-1 by myeloma cells suggesting that IL-6 may be indirectly promoting myeloma growth by increasing the recruitment of tumor-associated macrophages.

\section{T lymphocytes}

Tumor cells express tumor-associated antigens, which are presented in class I major histocompatibility complex molecules at the cell surface and can be processed by antigen-presenting cells for presentation in class II major histocompatibility complex molecules. Circulating naïve $\mathrm{T}$ cells encounter and recognize the major histocompatibility complex peptide complexes on the tumor cells and on the antigen-presenting cells. These interactions lead to the generation of increased numbers of CD8+ cytotoxic T lymphocytes that can destroy tumor tissue. To increase the numbers of tumor-infiltrating lymphocytes, adoptive-cell-transfer therapy has been used to remove tumor antigen-specific CD8+ cytotoxic $\mathrm{T}$ lymphocytes from patients, expand them ex vivo and reinfuse them back into the patient. ${ }^{68}$ Cole et al. ${ }^{56}$ recently showed that viruses could 'hitchhike' on antigen-specific $\mathrm{T}$ cells to reach and 'hand-off' therapeutic virus at tumor sites. However, some tumors do not release sufficient amounts of tumor-associated antigens to elicit a cytotoxic T-lymphocyte response. In another recent study, Qiao et al. ${ }^{69}$ took advantage of the trafficking pathway of naïve $\mathrm{T}$ cells, which home to lymph tissue due their CCR7 expression, to deliver VSV to draining lymph nodes and spleen thus preventing primary tumor metastasis. They illustrated that the adoptive transfer of $\mathrm{T}$ cells carrying VSV in mice bearing subcutaneous melanoma tumors was successful at preventing neometastatic growth in the draining lymph nodes and the spleen.

Thorne et al. ${ }^{70}$ exploited cytokine-induced killer (CIK) cell trafficking to deliver oncolytic vaccinia virus to sites of tumor growth. CIK cells are non-major histocompatibility complex-restricted tumor-killing cells that can be collected from patients and expanded in vitro. They destroy tumor cells by recognizing natural killer group $2 \mathrm{D}$ receptor, which is expressed on many tumor cells. ${ }^{71}$ The expression of natural killer group 2D increases in response to stress signals that are present in the tumor microenvironment or which result from virus infection. ${ }^{72}$ There was a modestly increased survival rate in mice bearing xenograft ovarian tumors treated with CIK cells or vaccinia virus alone. However, when the mice were treated with CIK cells infected with vaccinia virus, there was complete tumor regression and $100 \%$ survival rate. This combination therapy also increased the survival rate in mice bearing CIK resistant tumor xenografts.

Recent studies have begun to explore the efficacy of T-cell carriers for delivery of oncolytic viruses to sites of $\mathrm{MM}$ tumor growth in the presence of antiviral antibodies. In the case of measles virus, over $90 \%$ of American adults have protective titers of antimeasles antibodies. ${ }^{73}$ Ong et al. ${ }^{57}$ recently demonstrated that $\mathrm{T}$ cells collected from healthy donors were susceptible to $\mathrm{MV}$ infection. In vitro studies illustrated that MV-infected T cells were able to transfer MV infection by heterofusion with myeloma cells. In vivo, T cells infected with MV could efficiently deliver MV to sites of tumor growth in mice with myeloma. Significantly, the infected $\mathrm{T}$ cells were more efficient than naked measles virions at reaching sites of MM tumor growth in mice that were passively immunized with measles antiserum. 

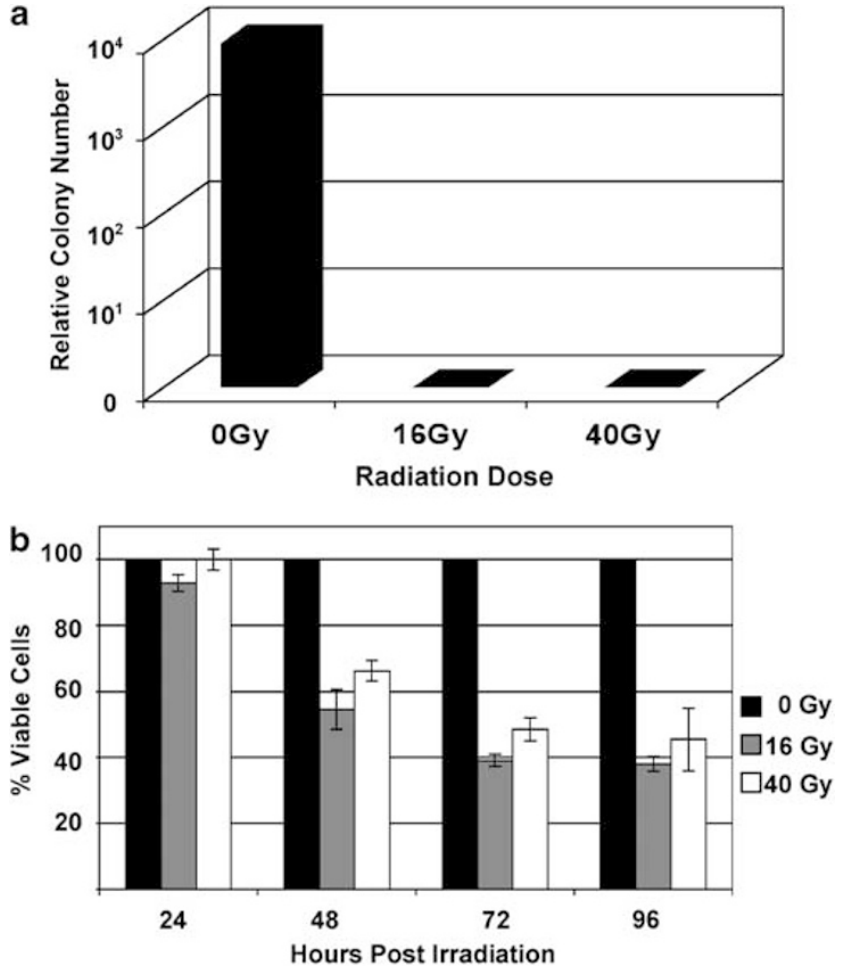

Figure 2 Colony-forming ability and cellular proliferation of irradiated 5TGM1 cells. (a) 5TGM1 cells $\left(7 \times 10^{4}\right)$ were treated with 0,16 or $40 \mathrm{~Gy}$ of $\gamma$-irradiation. Irradiation was performed at room temperature with a ${ }^{137} \mathrm{Cs}$ unit (JL Shepherd, Mark I, Model 25, CA, USA) on a rotating platform at a dose of $0.5 \mathrm{~Gy} \mathrm{~min}^{-1}$. Clonogenic assays were read on day 14 when cell colonies consisting of $>50$ cells were counted and expressed relative to the colony number in the untreated sample. (b) The 5TGM1 cells were irradiated as described above. At various times after irradiation, a 3-[4,5]dimethylthiazol2,5-diphenyltetrazolium bromide cell viability assay was performed following manufacturer's instructions (Cell Proliferation Kit I, Roche, Indianapolis, IN, USA). The data are presented as percent cell viability (OD of the test sample/OD of the control $\times 100$ ). Each value represents a mean \pm s.d. of three independent experiments.

\section{Myeloma cells}

Primary myeloma cells are derived from antibodysecreting plasma cells. The development of $\mathrm{B}$ cells into plasma cells has been well characterized and it has been shown that chemokine receptors and their ligands are major determinants of the changing homing properties of these cells as they pass through different stages of maturation. Naïve B cells develop from hematopoietic stem cells in the bone marrow, migrate to lymph nodes, encounter antigen, mature into plasma cells and the plasma cells return back to the bone marrow. ${ }^{74}$ Migration of B cells to the lymph nodes is dependent upon increased expression of the chemokine receptor CCR7. The ligands for CCR7 are CCL19 and CCL21, which are abundantly expressed in lymphatic tissue. ${ }^{15,76}$ Once in the lymph nodes, the antigen-activated $\mathrm{B}$ cells proliferate in the germinal centers. ${ }^{77}$ The germinal center contains two compartments termed light and dark zones and the migration of $\mathrm{B}$ cells between the two zones is dependent upon a chemokine expression gradient. B cells proliferate in the dark zone and interact with their target antigen in the light zone. The light zone contains many processes a
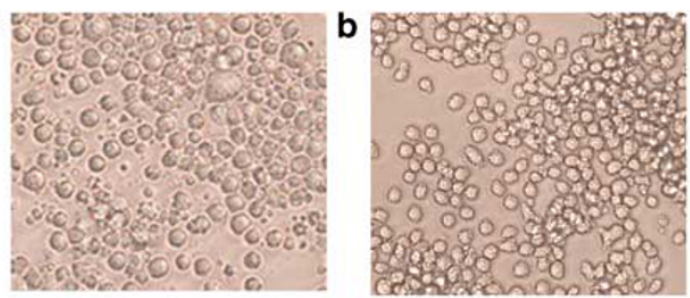

C
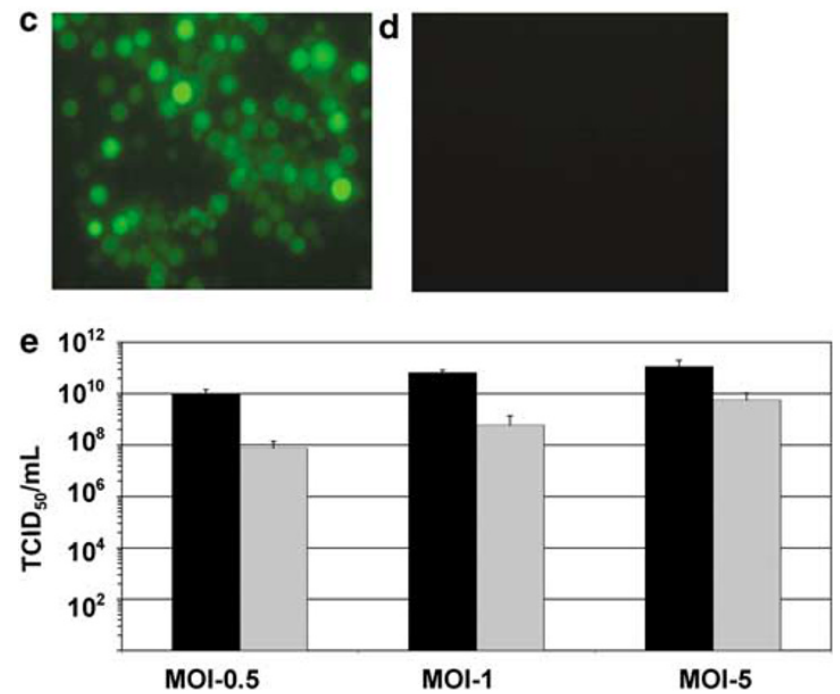

Figure 3 Irradiated 5TGM1 cells can be efficiently infected with VSV-GFP and can propagate the virus. (a, c) Irradiated (40 Gy) cells were infected with VSV-GFP $(\mathrm{MOI}=5)$. Twelve hours after infection, the cells were photographed under (a) phase contrast and (c) blue FITC filter. (b, d) Uninfected irradiated 5TGM1 cells were also photographed under the (b) phase contrast and (d) blue FITC filter. (e) Irradiated 5TGM1 cells were infected with VSV-GFP (MOI $=0.5,1$ and 5) for $30 \mathrm{~min}$ at $37^{\circ} \mathrm{C}$, supernatants were harvested at $48 \mathrm{~h}$ after infection and virus titers (TCID50 per ml) were determined on Vero cells. Each value represents a mean \pm s.d. of three independent experiments. FITC, fluorescein isothiocyanate; GFP, green fluorescent protein; MOI, multiplicity of infection; VSV, vesicular stomatitis virus.

from the follicular dendritic cell network and B cells pick up antigen here. B-cell progeny emerging from the dark zone express abundant CXCR5 and therefore polarize to the light zone where the chemokine CCL13 (its receptor is CXCR5) is highly expressed in the follicular dendritic cells. Once B cells encounter antigen, their expression of CXCR4 is quickly increased. CXCL12, the ligand for CXCR4, is expressed at a higher concentration in the dark zone than in the light zone of the germinal centers. Therefore, the B cells successfully competing for access to antigen in the light zone migrate to the dark zone for another round of replication. ${ }^{78,79}$ Before $\mathrm{B}$ cells exit the germinal centers, they lose their expression of CCR7. Subsequently, they rapidly differentiate into long-lived plasma cells or memory B cells, their increased CXCR4 expression allowing them to return to the bone marrow, where CXCL12 expression is abundant. ${ }^{80,81}$

Myeloma plasma cells are derived from normal plasma cells and therefore exhibit similar homing properties. Thus, primary myeloma cells are negative for CCL7, express abundant CXCR4 and home efficiently to the bone marrow. ${ }^{82,83}$ 

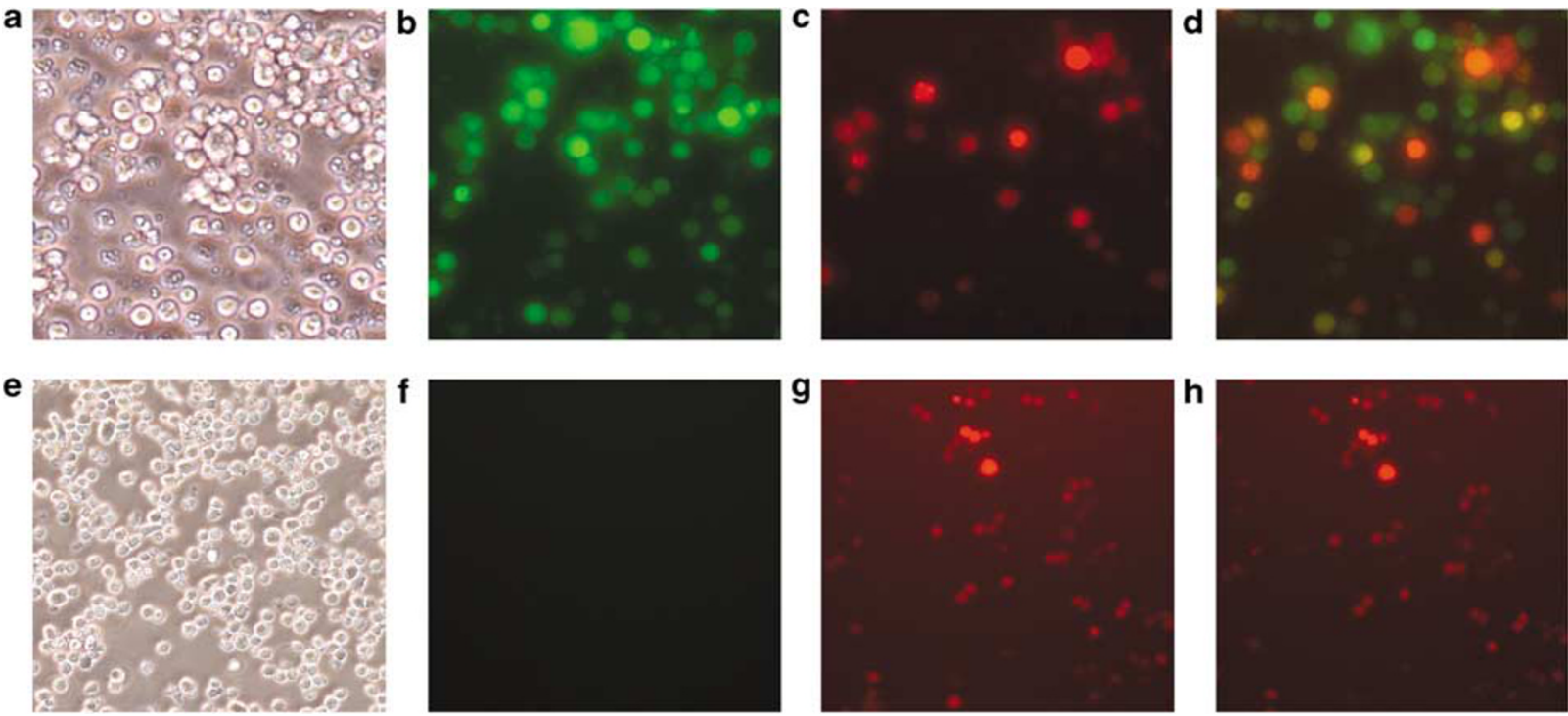

Figure 4 Transfer of VSV-GFP infection from irradiated 5TGM1 cells to uninfected 5TGM1 cells. (a-d) Infected, irradiated cells $\left(1 \times 10^{6}\right)$ were infected with VSV-GFP $(\mathrm{MOI}=5)$ and mixed with cherry red irradiated 5TGM1 cells $\left(1 \times 10^{6}\right)$. Micrographs were taken at $\times 20$ magnification under (a) phase contrast, (b) blue FITC filter, (c) Texas Red filters and (d) dual filter. (e-h) As a control, uninfected irradiated cells were mixed with cherryred irradiated 5TGM1 cells as described above. The control cells were photographed under (a) phase contrast, (b) blue FITC filter, (c) Texas Red filter and (d) dual filter. FITC, fluorescein isothiocyanate; GFP, green fluorescent protein; MOI, multiplicity of infection; VSV, vesicular stomatitis virus.

\section{Feasibility study of myeloma cells as carriers for oncolytic virus delivery}

Myeloma cells have an increased expression of CXCR4 and home to sites of MM tumor growth in the bone marrow. Also, tumor cells from histologically similar tumors are known to preferentially bind with one another, ${ }^{49}$ which might be expected to aid in the transfer of oncolytic virus infection from a myeloma cell carrier to uninfected myeloma cells. We therefore performed experiments to evaluate the use of MM cells as cell carriers for the treatment of $\mathrm{MM}$ with oncolytic viruses. While primary myeloma plasma cells are readily available from the bone marrow, they cannot be expanded ex vivo and reinfused back into the patient, which is a significant limitation. It would be considerably more convenient to use a myeloma cell line that is readily available at all times. We, therefore, sought to determine whether myeloma cell lines can provide a suitable carrier for the delivery of oncolytic VSV to sites of myeloma tumor growth.

The 5TGM1 murine myeloma cell line utilized in this present study is a variant of the parental 5T murine myeloma, which originated in C57BL/KaLwRij mice that spontaneously develop MM. ${ }^{84}$ In order for tumor cells to be administered into human patients, the cells must be exposed to ionizing radiation. The FDA has previously mandated a dose of $40 \mathrm{~Gy}$ to prevent cell proliferation with certainty, thus eliminating the possibility of neometastatic growth. Studies have shown that tumor cells exposed to 15-45 Gy of $\gamma$-radiation could still be used as carriers for in vivo delivery of oncolytic herpes virus and parvoiruses. ${ }^{49,52}$

\section{Susceptibility of 5TGM1 myeloma cells to ionizing radiation}

To determine whether 40 Gy of $\gamma$-radiation was sufficient to destroy the clonogenicity of 5TGM1 cells, the cells were exposed to 16 and $40 \mathrm{~Gy}$ of ionizing radiation and a methylcellulose clonogenic assay was utilized to determine cell proliferation. Directly after irradiation the cells $\left(7 \times 10^{4}\right)$ were washed with phosphate-buffered saline (PBS) and resuspended in $0.5 \mathrm{ml}$ of Iscove-modified Dulbecco medium supplemented with $10 \%$ fetal bovine serum and penicillin-streptomycin antibiotics. The $0.5 \mathrm{ml}$ cell suspension was added to $2.5 \mathrm{ml} 1.2 \%$ methylcellulose medium and the mixtures were plated in $35-\mathrm{mm}$ Petri dishes and maintained at $37^{\circ} \mathrm{C}$ and $5 \% \mathrm{CO}_{2}$. After 14 days incubation, colonies that contained greater than 50 cells were counted in both irradiated and nonirradiated cell populations. As illustrated in Figure 2a, 16 or 40 Gy ionizing radiation completely inhibited the colony formation of 5TGM1 cells. Despite the accumulation of lethal DNA mutations that destroy clonogenicity, irradiated cells may remain metabolically viable for long periods of time. Viability of the irradiated cells was therefore analyzed at various times post-irradiation using a 3-[4,5]dimethylthiazol-2,5-diphenyltetrazolium bromide cell viability assay. At $24 \mathrm{~h}$ after irradiation, almost $100 \%$ of the cells were still viable (Figure 2b). Interestingly, at $96 \mathrm{~h}$ after irradiation, about $40-50 \%$ of irradiated cells remained viable. These results show that although the irradiated myeloma 5TGM1 cells no longer proliferate, they still maintain their metabolic viability for at least 4 days after they have been irradiated.

\section{Susceptibility of irradiated 5TGM1 cells to VSV-GFP infection}

To determine susceptibility to VSV-green fluorescent protein (GFP) infection, the irradiated cells were infected with VSV-GFP (multiplicity of infection $(\mathrm{MOI})=5$ ). Immediately following irradiation virus was added to the cells for $30 \mathrm{~min}$ at $37^{\circ} \mathrm{C}$, free virus was then removed and the cells were washed with PBS and resuspended in fresh Iscove-modified Dulbecco complete medium. At $12 \mathrm{~h}$ after infection, the infected cells were photographed 
using a fluorescence microscope. As illustrated in Figures $3 \mathrm{a}$ and $\mathrm{c}$, at $12 \mathrm{~h}$ after infection, the VSV-GFP exposed myeloma cells were strongly GFP positive indicating that they had been efficiently infected with VSV-GFP. In addition, we were not able to detect GFP-positive cells in uninfected 5TGM1 population (Figures $3 b$ and $d$ ). Similar results were obtained when the irradiated cells were infected with VSV-GFP $(\mathrm{MOI}=0.5$ and 1$)$ (data not shown).

We next sought to determine whether irradiated 5TGM1 cells could release VSV-GFP progeny after they had been infected. Briefly, irradiated and non-irradiated cells were infected with VSV-GFP $(\mathrm{MOI}=0.5,1$ and 5$)$ as described previously. At $48 \mathrm{~h}$ after infection, culture supernatants from infected cells were collected and virus titers were determined on Vero cells using TCID $_{50}$ titration method. As depicted in Figure 3e, cells exposed to $40 \mathrm{~Gy}$ were still able to produce significant amounts of VSV-GFP, although there was a slight decrease in progeny virus production compared to non-irradiated cells.

\section{Virus transfer from VSV-infected 5TGM1 carriers to uninfected myeloma cells}

Myeloma cells infected with VSV-GFP were co-cultured with target uninfected myeloma cells to determine if VSV-GFP infection could be transferred. To visualize the targeted cells, we generated 5TGM1 expressing the cherry red reporter gene (5TGM1-Cherry) using a lentivector expression system. Briefly, irradiated (40 Gy) 5TGM1 cells were infected with VSV-GFP $(\mathrm{MOI}=5)$ as described above. At $4 \mathrm{~h}$ after infection, the cells were washed twice in PBS and mixed with uninfected 5TGM1Cherry cells in a 1:1 ratio. As illustrated in Figure 4d, efficient transfer of VSV infection resulted in cells that were both GFP and cherry red positive.

\section{In vivo trafficking and antitumor activity of VSV-infected 5TGM1-Luc cells}

Since the results of our in vitro studies seemed promising, we next evaluated the in vivo trafficking of VSVinfected myeloma cells. In this pilot myeloma cell trafficking study, we used an orthotopic human myeloma model where irradiated (150 cGy) female non-obese diabetic/severe-combined immunodeficiency mice were challenged intravenously with $10^{7}$ KAS/61 cells. Human myeloma paraprotein (IgG- $\kappa$ ) levels were measured in blood samples from these mice to monitor myeloma progression. On day 65, mice with detectable levels of IgG $-\kappa$ were randomized into control and treatment groups. The control group $(n=2)$ received one administration of irradiated (40 Gy) $5 \times 10^{6} 5$ TGM1-Luc cells via the tail vein. To monitor the cells in the mice after systemic administration, they were transduced with luciferase reporter gene using a lentivector expression system. The treated group $(n=3)$ were given $5 \times 10^{6}$ of VSV-infected 5TGM1-Luc cells systemically. Briefly, the irradiated (40 Gy) 5TGM1-Luc cells were infected with VSV-GFP (MOI=5.0). Four hours after infection, the cells were washed three times in PBS and then introduced into mice via the tail vein. The Xenogen IVIS 200 Bioluminescence system was utilized to detect luciferase activity from the 5TGM1-Luc cells. Briefly, mice were injected intraperitoneally with D-luciferin and then were

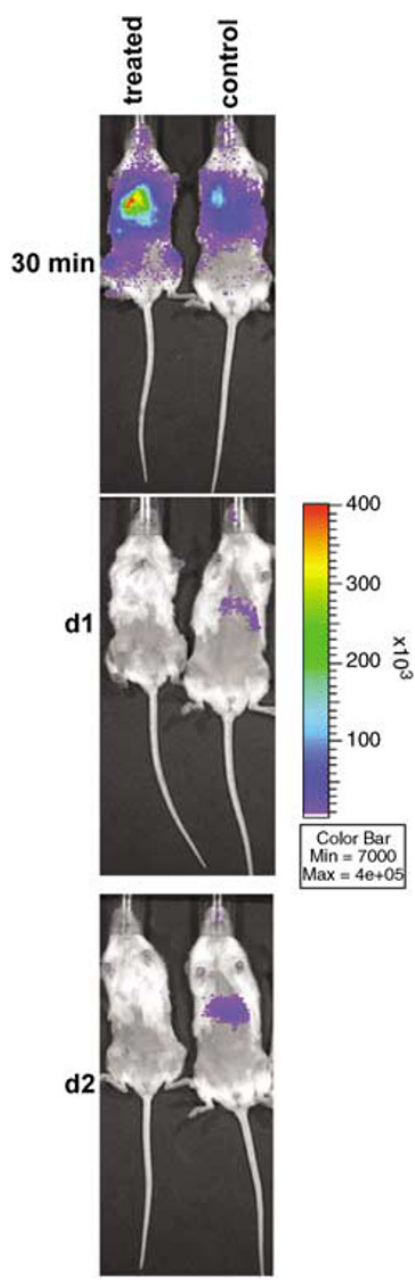

Figure 5 Trafficking of VSV-GFP-infected cells in orthotopic myeloma tumors in mice. Non-obese diabetic/severe-combined immunodeficiency mice with disseminated myeloma were infused intravenously with irradiated 5TGM1-Luc cells $\left(5 \times 10^{6}\right)$ preinfected with VSV-GFP $(\mathrm{MOI}=5)$ or only with 5TGM1-Luc cells $\left(5 \times 10^{6}\right)$, at 9 weeks post-myeloma cell implantation via tail-vein injection. Bioluminescent imaging to detect the 5TGM1-Luc signal in mice was performed at $30 \mathrm{~min}, 24$ and $48 \mathrm{~h}$ after cell therapy. The level of cellular firefly luciferase is expressed as photons per second per $\mathrm{cm}^{2}$. GFP, green fluorescent protein; MOI, multiplicity of infection; VSV, vesicular stomatitis virus.

anesthetized via isoflurane inhalation. Mice were then placed on the imaging platform and images were taken within $5 \mathrm{~min}$ after injection of luciferin. As illustrated in Figure 5, both the VSV-infected 5TGM1-Luc and uninfected 5TGM1-Luc cells were detected in the lungs at $30 \mathrm{~min}$ after infusion. However, at $24 \mathrm{~h}$ after administration, luciferase activity was no longer detectable in lung, but was instead detected in the liver. There was no detectable luciferase signal in mice that received VSVinfected cells at the 24- or 48-h time points, suggesting that the VSV-infected cells had been killed by the VSV infection.

Six days after 5TGM1 cells were injected, we collected serum samples from both the control and treated groups and measured human IgG- $\kappa$ levels. As illustrated in Figure 6, in mice that received only 5TGM1-Luc cells, there was an increase in human IgG- $\kappa$ levels, indicating that the myeloma progressed. However, in mice that 
804

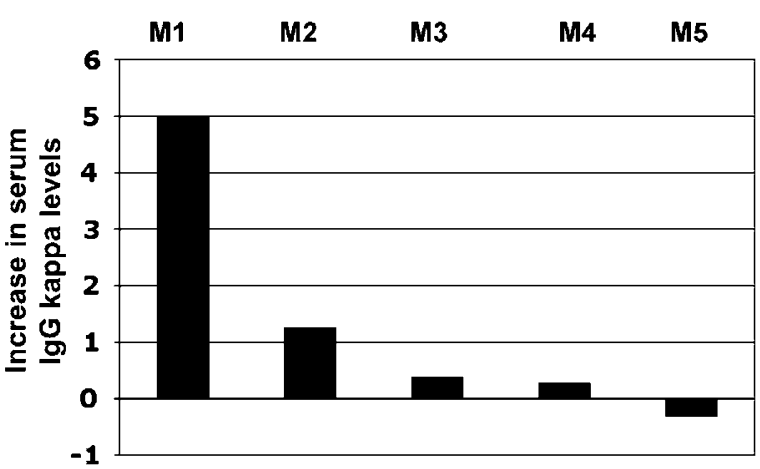

Figure 6 The antitumor activity of VSV-GFP infection in an orthotopic mouse myeloma model after systemic cell-mediated delivery of the virus. Myeloma burden of control (M1 and M2) and treatment (M3, M4, M5) groups was determined by measuring human IgG- $\kappa$ levels in serum at baseline and on day 6 after treatment. Bar graph illustrating the change in serum IgG- $\kappa$ levels during the treatment period in each mouse. GFP, green fluorescent protein; VSV, vesicular stomatitis virus.

received VSV-infected 5TGM1-Luc cells, there was only a small increase, or in one mouse a decrease in human IgG- $\kappa$ levels, which indicated a decrease in myeloma progression. To study the trafficking of VSV-infected 5TGM1-Luc cells, on day 6 after treatment, the mice were killed and cells from the spleen were harvested, washed three times in PBS and overlaid onto Vero cell monolayers in a 1:1 ratio. The following day, the cell monolayers were examined under fluorescence microscopy to detect GFP-positive cells, which would indicate the presence of VSV-GFP. The spleen is a hematopoietic organ in mice and myeloma cells have been show to localize in both spleen and bone marrow in mouse models. ${ }^{85}$ In two out of three treated mice, we were able to recover VSV-GFP from spleen tissue (data not shown). As expected, VSV was not recovered from the control group animals (data not shown). Thus, the irradiated, VSV-infected 5TGM1 myeloma cells were able to deliver replication competent VSV to sites of myeloma tumor growth.

\section{Conclusion}

Multiple myeloma is a disseminated, incurable disease that offers an excellent paradigm for systemic oncolytic virus therapy. There have been several studies that have demonstrated the promise of oncolytic viruses as a novel approach to treat MM. Since, MM is a disseminated disease, the oncolytic viruses must be delivered via the bloodstream. There are many barriers that prevent intravenously delivered oncolytic viruses from efficiently reaching the sites of tumor growth and destroying the tumor. To address these barriers, recent studies used cell carriers to deliver oncolytic virus to sites of tumor growth. In this review, we discussed four cell types (tumor cells, T cells, monocytes and mesenchymal progenitor cells) that can traffic to the bone marrow, the major site of myeloma growth, and that should be considered as candidates to deliver oncolytic viruses to the sites of myeloma growth. We performed feasibility studies demonstrating that MM cells can be used to deliver VSV-GFP to the sites of myeloma tumor growth. Our results indicate that murine 5TGM1 myeloma cells can be irradiated to destroy clonogenicity, but that the irradiated cells retain their metabolic viability for at least 4 days thereafter. In addition, the irradiated 5TGM1 cells remained susceptible to VSV-GFP infection and were able to produce VSV-GFP progeny at levels similar to unirradiated cells. In a pilot myeloma cell trafficking study, we used an orthotopic human myeloma model and delivered VSV-GFP-infected myeloma cells systemically. Our results are still preliminary but they do show that VSV-GFP-infected myeloma cells can be used to deliver VSV-GFP intravenously to the sites of myeloma tumor growth. Based on the preliminary results we presented in this review, we are performing additional studies to determine the optimal cell carrier for the treatment of MM.

\section{References}

1 Kyle RA. Multiple myeloma: an odyssey of discovery. Br J Haematol 2000; 111: 1035-1044.

2 Angel JB, Walpita P, Lerch RA, Sidhu MS, Masurekar M, DeLellis RA et al. Vaccine-associated measles pneumonitis in an adult with AIDS. Ann Intern Med 1998; 129: 104-106.

3 Bjorkstrand B. European Group for Blood and Marrow Transplantation Registry studies in multiple myeloma. Semin Hematol 2001; 38: 219-225.

4 Fassas A, Tricot G. Results of high-dose treatment with autologous stem cell support in patients with multiple myeloma. Semin Hematol 2001; 38: 231-242.

5 Kyle RA, Rajkumar SV. Multiple myeloma. N Engl J Med 2004; 351: 1860-1873.

6 Hideshima T, Mitsiades C, Tonon G, Richardson PG, Anderson $\mathrm{KC}$. Understanding multiple myeloma pathogenesis in the bone marrow to identify new therapeutic targets. Nat Rev Cancer 2007; 7: 585-598.

7 Alsayed Y, Ngo H, Runnels J, Leleu X, Singha UK, Pitsillides CM et al. Mechanisms of regulation of CXCR4/SDF-1 (CXCL12)dependent migration and homing in multiple myeloma. Blood 2007; 109: 2708-2717.

8 De Clercq E. Potential clinical applications of the CXCR4 antagonist bicyclam AMD3100. Mini Rev Med Chem 2005; 5: 805-824.

9 Hideshima T, Chauhan D, Hayashi T, Podar K, Akiyama M, Gupta D et al. The biological sequelae of stromal cell-derived factor-1alpha in multiple myeloma. Mol Cancer Ther 2002; 1: 539-544.

10 Akiyama M, Hideshima T, Hayashi T, Tai YT, Mitsiades CS, Mitsiades $\mathrm{N}$ et al. Cytokines modulate telomerase activity in a human multiple myeloma cell line. Cancer Res 2002; 62: 3876-3882.

11 Chauhan D, Li G, Hideshima T, Podar K, Shringarpure R, Mitsiades $C$ et al. Blockade of ubiquitin-conjugating enzyme CDC34 enhances anti-myeloma activity of Bortezomib/Proteasome inhibitor PS-341. Oncogene 2004; 23: 3597-3602.

12 Damiano JS, Cress AE, Hazlehurst LA, Shtil AA, Dalton WS. Cell adhesion mediated drug resistance (CAM-DR): role of integrins and resistance to apoptosis in human myeloma cell lines. Blood 1999; 93: 1658-1667.

13 Hideshima T, Catley L, Yasui H, Ishitsuka K, Raje N, Mitsiades C et al. Perifosine, an oral bioactive novel alkylphospholipid, inhibits Akt and induces in vitro and in vivo cytotoxicity in human multiple myeloma cells. Blood 2006; 107: 4053-4062.

14 Mitsiades CS, Mitsiades NS, Munshi NC, Richardson PG, Anderson $\mathrm{KC}$. The role of the bone microenvironment in the 
pathophysiology and therapeutic management of multiple myeloma: interplay of growth factors, their receptors and stromal interactions. Eur J Cancer 2006; 42: 1564-1573.

15 Hallek M, Bergsagel PL, Anderson KC. Multiple myeloma: increasing evidence for a multistep transformation process. Blood 1998; 91: 3-21.

16 Richardson PG, Barlogie B, Berenson J, Singhal S, Jagannath S, Irwin $\mathrm{D}$ et al. A phase 2 study of bortezomib in relapsed, refractory myeloma. N Engl J Med 2003; 348: 2609-2617.

17 Richardson PG, Schlossman RL, Weller E, Hideshima T, Mitsiades C, Davies F et al. Immunomodulatory drug CC-5013 overcomes drug resistance and is well tolerated in patients with relapsed multiple myeloma. Blood 2002; 100: 3063-3067.

18 Richardson PG, Sonneveld P, Schuster MW, Irwin D, Stadtmauer $\mathrm{EA}$, Facon $\mathrm{T}$ et al. Bortezomib or high-dose dexamethasone for relapsed multiple myeloma. $N$ Engl J Med 2005; 352: 2487-2498.

19 Singhal S, Mehta J, Desikan R, Ayers D, Roberson P, Eddlemon P et al. Antitumor activity of thalidomide in refractory multiple myeloma. N Engl J Med 1999; 341: 1565-1571.

20 Jemal A, Siegel R, Ward E, Murray T, Xu J, Thun MJ. Cancer statistics, 2007. CA Cancer J Clin 2007; 57: 43-66.

21 Parato KA, Senger D, Forsyth PA, Bell JC. Recent progress in the battle between oncolytic viruses and tumours. Nat Rev Cancer 2005; 5: 965-976.

22 Kelly E, Russell SJ. History of oncolytic viruses: genesis to genetic engineering. Mol Ther 2007; 15: 651-659.

23 Nakamura T, Russell SJ. Oncolytic measles viruses for cancer therapy. Expert Opin Biol Ther 2004; 4: 1685-1692.

24 Peng KW, Ahmann GJ, Pham L, Greipp PR, Cattaneo R, Russell SJ. Systemic therapy of myeloma xenografts by an attenuated measles virus. Blood 2001; 98: 2002-2007.

25 Dorig RE, Marcil A, Chopra A, Richardson CD. The human CD46 molecule is a receptor for measles virus (Edmonston strain). Cell 1993; 75: 295-305.

26 Hsu EC, Iorio C, Sarangi F, Khine AA, Richardson CD. CDw150 (SLAM) is a receptor for a lymphotropic strain of measles virus and may account for the immunosuppressive properties of this virus. Virology 2001; 279: 9-21.

27 Peng KW, Donovan KA, Schneider U, Cattaneo R, Lust JA, Russell SJ. Oncolytic measles viruses displaying a single-chain antibody against CD38, a myeloma cell marker. Blood 2003; 101: 2557-2562.

28 Fishelson Z, Donin N, Zell S, Schultz S, Kirschfink M. Obstacles to cancer immunotherapy: expression of membrane complement regulatory proteins (mCRPs) in tumors. Mol Immunol 2003; 40: 109-123.

29 Anderson BD, Nakamura T, Russell SJ, Peng KW. High CD46 receptor density determines preferential killing of tumor cells by oncolytic measles virus. Cancer Res 2004; 64: 4919-4926.

30 Ong HT, Timm MM, Greipp PR, Witzig TE, Dispenzieri A, Russell SJ et al. Oncolytic measles virus targets high CD46 expression on multiple myeloma cells. Exp Hematol 2006; 34: 713-720.

31 Cho JY, Shen DH, Yang W, Williams B, Buckwalter TL, La Perle $\mathrm{KM}$ et al. In vivo imaging and radioiodine therapy following sodium iodide symporter gene transfer in animal model of intracerebral gliomas. Gene Therapy 2002; 9: 1139-1145.

32 Spitzweg C, O'Connor MK, Bergert ER, Tindall DJ, Young CY, Morris JC. Treatment of prostate cancer by radioiodine therapy after tissue-specific expression of the sodium iodide symporter. Cancer Res 2000; 60: 6526-6530.

33 Dingli D, Peng KW, Harvey ME, Greipp PR, O'Connor MK, Cattaneo $\mathrm{R}$ et al. Image-guided radiovirotherapy for multiple myeloma using a recombinant measles virus expressing the thyroidal sodium iodide symporter. Blood 2004; 103: 1641-1646.

34 Myers RM, Greiner SM, Harvey ME, Griesmann G, Kuffel MJ, Buhrow SA et al. Preclinical pharmacology and toxicology of intravenous MV-NIS, an oncolytic measles virus administered with or without cyclophosphamide. Clin Pharmacol Ther 2007; 82: 700-710.

35 Belkowski LS, Sen GC. Inhibition of vesicular stomatitis viral mRNA synthesis by interferons. J Virol 1987; 61: 653-660.

36 Stojdl DF, Lichty B, Knowles S, Marius R, Atkins H, Sonenberg N et al. Exploiting tumor-specific defects in the interferon pathway with a previously unknown oncolytic virus. Nature medicine 2000; 6: 821-825.

37 Obuchi M, Fernandez M, Barber GN. Development of recombinant vesicular stomatitis viruses that exploit defects in host defense to augment specific oncolytic activity. J Virol 2003; 77: 8843-8856.

38 Stojdl DF, Lichty BD, tenOever BR, Paterson JM, Power AT, Knowles $\mathrm{S}$ et al. VSV strains with defects in their ability to shutdown innate immunity are potent systemic anti-cancer agents. Cancer Cell 2003; 4: 263-275.

39 Lichty BD, Stojdl DF, Taylor RA, Miller L, Frenkel I, Atkins H et al. Vesicular stomatitis virus: a potential therapeutic virus for the treatment of hematologic malignancy. Hum Gene Ther 2004; 15: 821-831.

40 Goel A, Carlson SK, Classic KL, Greiner S, Naik S, Power AT et al. Radioiodide imaging and radiovirotherapy of multiple myeloma using VSV(Delta51)-NIS, an attenuated vesicular stomatitis virus encoding the sodium iodide symporter gene. Blood 2007; 110: 2342-2350.

41 Lun X, Senger DL, Alain T, Oprea A, Parato K, Stojdl D et al. Effects of intravenously administered recombinant vesicular stomatitis virus (VSV(deltaM51)) on multifocal and invasive gliomas. Journal of the National Cancer Institute 2006; 98: 1546-1557.

42 Pallansch MAaRRP. Enteroviruses: polioviruses, coxsackieviruses, echoviruses, and newer enteroviruses. In: Knipe PMH DM, Griffin DE, Lamb RA, Martin MA, Roizman B, Straus SE (eds). Fields Virology. Lippincott Williams \& Wilkins: Philadelphia, PA, 2001, pp 723-776.

$43 \mathrm{Au}$ GG, Lindberg AM, Barry RD, Shafren DR. Oncolysis of vascular malignant human melanoma tumors by Coxsackievirus A21. Int J Oncol 2005; 26: 1471-1476.

44 Shafren DR, Au GG, Nguyen T, Newcombe NG, Haley ES, Beagley $L$ et al. Systemic therapy of malignant human melanoma tumors by a common cold-producing enterovirus, coxsackievirus a21. Clin Cancer Res 2004; 10: 53-60.

45 Shafren DR, Dorahy DJ, Ingham RA, Burns GF, Barry RD. Coxsackievirus A21 binds to decay-accelerating factor but requires intercellular adhesion molecule 1 for cell entry. J Virol 1997; 71: 4736-4743.

46 Au GG, Lincz LF, Enno A, Shafren DR. Oncolytic Coxsackievirus A21 as a novel therapy for multiple myeloma. $\mathrm{Br}$ J Haematol 2007; 137: 133-141.

47 Kawa AaA S. The effect of attenuated vaccinia virus AS strain on multiple myeloma; a case report. Japan Journal of Experimental Medicine 1987; 57: 79-81.

48 Arakawa Jr S, Hamami G, Umezu K, Kamidono S, Ishigami J, Arakawa S. Clinical trial of attenuated vaccinia virus AS strain in the treatment of advanced adenocarcinoma. Report on two cases. J Cancer Res Clin Oncol 1987; 113: 95-98.

49 Coukos G, Makrigiannakis A, Kang EH, Caparelli D, Benjamin I, Kaiser LR et al. Use of carrier cells to deliver a replicationselective herpes simplex virus-1 mutant for the intraperitoneal therapy of epithelial ovarian cancer. Clin Cancer Res 1999; 5: 1523-1537.

50 Garcia-Castro J, Martinez-Palacio J, Lillo R, Garcia-Sanchez F, Alemany R, Madero L et al. Tumor cells as cellular vehicles to deliver gene therapies to metastatic tumors. Cancer Gene Ther 2005; 12: 341-349.

51 Power AT, Bell JC. Cell-based delivery of oncolytic viruses: a new strategic alliance for a biological strike against cancer. Mol Ther 2007; 15: 660-665. 
52 Raykov Z, Balboni G, Aprahamian M, Rommelaere J. Carrier cell-mediated delivery of oncolytic parvoviruses for targeting metastases. Int J Cancer 2004; 109: 742-749.

53 Jevremovic D, Gulati R, Hennig I, Diaz RM, Cole C, Kleppe L et al. Use of blood outgrowth endothelial cells as virusproducing vectors for gene delivery to tumors. Am J Physiol Heart Circ Physiol 2004; 287: H494-H500.

54 Hakkarainen T, Sarkioja M, Lehenkari P, Miettinen S, Ylikomi T, Suuronen $\mathrm{R}$ et al. Human mesenchymal stem cells lack tumor tropism but enhance the antitumor activity of oncolytic adenoviruses in orthotopic lung and breast tumors. Hum Gene Ther 2007; 18: 627-641.

55 Komarova S, Kawakami Y, Stoff-Khalili MA, Curiel DT, Pereboeva L. Mesenchymal progenitor cells as cellular vehicles for delivery of oncolytic adenoviruses. Mol Cancer Ther 2006; 5: 755-766.

56 Cole C, Qiao J, Kottke T, Diaz RM, Ahmed A, Sanchez-Perez L et al. Tumor-targeted, systemic delivery of therapeutic viral vectors using hitchhiking on antigen-specific $\mathrm{T}$ cells. Nature medicine 2005; 11: 1073-1081.

57 Ong HT, Hasegawa K, Dietz AB, Russell SJ, Peng KW. Evaluation of T cells as carriers for systemic measles virotherapy in the presence of antiviral antibodies. Gene Therapy 2007; 14: 324-333.

58 Iankov ID, Blechacz B, Liu C, Schmeckpeper JD, Tarara JE, Federspiel MJ et al. Infected cell carriers: a new strategy for systemic delivery of oncolytic measles viruses in cancer virotherapy. Mol Ther 2007; 15: 114-122.

59 Prockop DJ. Marrow stromal cells as stem cells for nonhematopoietic tissues. Science 1997; 276: 71-74.

60 Studeny M, Marini FC, Dembinski JL, Zompetta C, CabreiraHansen M, Bekele BN et al. Mesenchymal stem cells: potential precursors for tumor stroma and targeted-delivery vehicles for anticancer agents. Journal of the National Cancer Institute 2004; 96: 1593-1603.

61 Pereboeva L, Komarova S, Mikheeva G, Krasnykh V, Curiel DT. Approaches to utilize mesenchymal progenitor cells as cellular vehicles. Stem Cells 2003; 21: 389-404.

62 Tacke F, Randolph GJ. Migratory fate and differentiation of blood monocyte subsets. Immunobiology 2006; 211: 609-618.

63 Jin H, Su J, Garmy-Susini B, Kleeman J, Varner J. Integrin alpha4beta1 promotes monocyte trafficking and angiogenesis in tumors. Cancer Res 2006; 66: 2146-2152.

64 Rinaldo Jr CR, Piazza P. Virus infection of dendritic cells: portal for host invasion and host defense. Trends Microbiol 2004; 12: 337-345.

65 Mantovani A, Sozzani S, Locati M, Allavena P, Sica A. Macrophage polarization: tumor-associated macrophages as a paradigm for polarized M2 mononuclear phagocytes. Trends Immunol 2002; 23: 549-555.

66 Sica A, Schioppa T, Mantovani A, Allavena P. Tumour-associated macrophages are a distinct M2 polarised population promoting tumour progression: potential targets of anti-cancer therapy. Eur J Cancer 2006; 42: 717-727.

67 Dankbar B, Padro T, Leo R, Feldmann B, Kropff M, Mesters RM et al. Vascular endothelial growth factor and interleukin-6 in paracrine tumor-stromal cell interactions in multiple myeloma. Blood 2000; 95: 2630-2636.

68 Dudley ME, Rosenberg SA. Adoptive-cell-transfer therapy for the treatment of patients with cancer. Nat Rev Cancer 2003; 3: 666-675.

69 Qiao J, Kottke T, Willmon C, Galivo F, Wongthida P, Diaz RM et al. Purging metastases in lymphoid organs using a combination of antigen-nonspecific adoptive $\mathrm{T}$ cell therapy, oncolytic virotherapy and immunotherapy. Nature medicine 2008; 14: 37-44.

70 Thorne SH, Negrin RS, Contag CH. Synergistic antitumor effects of immune cell-viral biotherapy. Science 2006; 311: 1780-1784.

71 Verneris MR, Karami M, Baker J, Jayaswal A, Negrin RS. Role of NKG2D signaling in the cytotoxicity of activated and expanded CD8+ T cells. Blood 2004; 103: 3065-3072.

72 Hamerman JA, Ogasawara K, Lanier LL. NK cells in innate immunity. Curr Opin Immunol 2005; 17: 29-35.

73 Audet S, Virata-Theimer ML, Beeler JA, Scott DE, Frazier DJ, Mikolajczyk MG et al. Measles-virus-neutralizing antibodies in intravenous immunoglobulins. J Infect Dis 2006; 194: 781-789.

74 Manz RA, Thiel A, Radbruch A. Lifetime of plasma cells in the bone marrow. Nature 1997; 388: 133-134.

75 Luther SA, Bidgol A, Hargreaves DC, Schmidt A, Xu Y, Paniyadi $\mathrm{J}$ et al. Differing activities of homeostatic chemokines CCL19, CCL21, and CXCL12 in lymphocyte and dendritic cell recruitment and lymphoid neogenesis. J Immunol 2002; 169: 424-433.

76 Okada T, Ngo VN, Ekland EH, Forster R, Lipp M, Littman DR et al. Chemokine requirements for B cell entry to lymph nodes and Peyer's patches. J Exp Med 2002; 196: 65-75.

77 Lui YJ, Zhang J, Lane PJ, Chan EY, MacLennan IC. Sites of specific $B$ cell activation in primary and secondary responses to T cell-dependent and T cell-independent antigens. Eur J Immunol 1991; 21: 2951-2962.

78 Allen CD, Ansel KM, Low C, Lesley R, Tamamura H, Fujii N et al. Germinal center dark and light zone organization is mediated by CXCR4 and CXCR5. Nat Immunol 2004; 5: 943-952.

79 Cyster JG, Ansel KM, Reif K, Ekland EH, Hyman PL, Tang HL et al. Follicular stromal cells and lymphocyte homing to follicles. Immunol Rev 2000; 176: 181-193.

80 Allen CD, Okada T, Cyster JG. Germinal-center organization and cellular dynamics. Immunity 2007; 27: 190-202.

81 Tokoyoda K, Egawa T, Sugiyama T, Choi BI, Nagasawa T. Cellular niches controlling B lymphocyte behavior within bone marrow during development. Immunity 2004; 20: 707-718.

82 Cyster JG. Homing of antibody secreting cells. Immunol Rev 2003; 194: 48-60.

83 Moller C, Stromberg T, Juremalm M, Nilsson K, Nilsson G. Expression and function of chemokine receptors in human multiple myeloma. Leukemia 2003; 17: 203-210.

84 Radl J CJ, Zurcher C, van den EndenVieveen MM, de Leeuw AM. Animal model of human disease: mulitple myeloma. Am J Cancer 1988; 132: 593-597.

85 Mori Y, Shimizu N, Dallas M, Niewolna M, Story B, Williams PJ et al. Anti-alpha4 integrin antibody suppresses the development of multiple myeloma and associated osteoclastic osteolysis. Blood 2004; 104: 2149-2154. 